Portland State University

PDXScholar

Spring 7-5-2018

\title{
Effects of Fan Cooling at Room Temperature on Indoor 20-km Cycling Time Trial Performance
}

Matthew Bloom

Portland State University

Follow this and additional works at: https://pdxscholar.library.pdx.edu/open_access_etds

Part of the Medicine and Health Sciences Commons

Let us know how access to this document benefits you.

Recommended Citation

Bloom, Matthew, "Effects of Fan Cooling at Room Temperature on Indoor 20-km Cycling Time Trial Performance" (2018). Dissertations and Theses. Paper 4482.

https://doi.org/10.15760/etd.6366

This Thesis is brought to you for free and open access. It has been accepted for inclusion in Dissertations and Theses by an authorized administrator of PDXScholar. Please contact us if we can make this document more accessible: pdxscholar@pdx.edu. 
Effects of Fan Cooling at Room Temperature on Indoor 20-km Cycling

Time Trial Performance

\author{
by \\ Matthew Bloom
}

A thesis submitted in partial fulfillment of the requirements for the degree of

Master of Science

in

Health Studies

Thesis Committee:

Gary Brodowicz, Chair

James Wallis

Randy Miller

Portland State University

2018 


\begin{abstract}
Aim: The primary purpose of this study was to examine effects of a fan-induced airflow at standard room temperature $\left(20-23^{\circ} \mathrm{C}\right)$ on $20-\mathrm{km}$ cycling time trial performance. A secondary purpose was to investigate two fan speeds and whether a difference in performance existed with increased fan speed in intermediate duration indoor cycling tests.
\end{abstract}

Methods: Seven trained cyclists completed three 20-km cycling time trials under three conditions in a randomized crossover design. The 3 conditions were: 1) control (no fan), 2) low speed fan, and 3) high speed fan.

Results: A tendency for modest decreases in time to completion (TTC) were noted in the two experimental conditions compared to the control condition $(-2.06 \%$ low speed fan; $-3.29 \%$ high speed fan). There was also a tendency for small increases in power output during the experimental conditions, although neither time nor power output differences were statistically significant. No differences in mean heart rate and rating of perceived exertion were observed among the three conditions.

Conclusion: These results suggest a relationship between fan use and 20-km indoor cycling time trial performance, specifically TTC and mean power output, but larger samples are needed to provide adequate statistical power. Further investigation into the effects of fan use in standard testing environments is recommended. No additional benefit was observed from increased fan speeds. Differences in the experimental conditions on average resulted in non-significant $1.2 \%$ improvement. 


\section{Table of Contents}

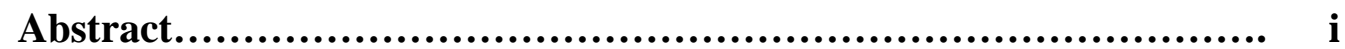

List of Figures............................................................ iii

Introduction........................................................... 1

Methods............................................................................. 5

Participants......................................................... 5

Procedures.................................................................... 5

Statistical Analysis................................................. 7

Results.............................................................................. 8

Discussion.......................................................... 14

Limitations......................................................... 18

Further Inquiry .................................................. 19

Conclusions............................................................ 21

References........................................................... 23 


\section{List of Figures}

Figure 1. Mean time to completion for each test condition $( \pm \mathrm{SD}) \ldots \ldots \ldots \ldots \ldots \ldots \ldots \ldots$

Figure 2. Time to completion across all conditions for all participants.................9

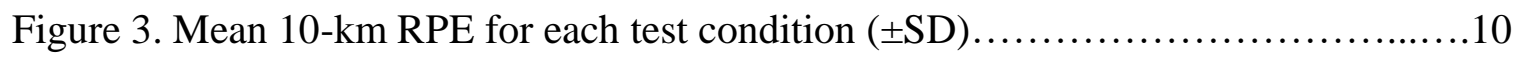

Figure 4. Mean 19-km RPE for each test condition $( \pm$ SD) $\ldots \ldots \ldots \ldots \ldots \ldots \ldots \ldots \ldots \ldots \ldots \ldots \ldots \ldots \ldots \ldots \ldots$

Figure 5. Mean power output for each test condition $( \pm \mathrm{SD}) \ldots \ldots \ldots \ldots \ldots \ldots \ldots \ldots \ldots \ldots \ldots \ldots \ldots \ldots$

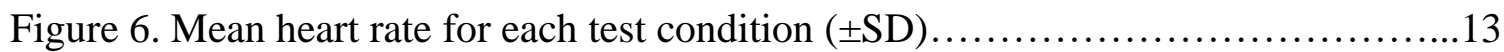




\section{Introduction}

Indoor exercise tests are regularly performed in academic or sports settings to determine fitness or measure performance. Coaches often conduct such tests to evaluate and track an athlete's training and fitness. One mode of testing that has become very popular in the sport of competitive cycling is Functional Threshold Power (FTP) testing, defined as the maximal power output that can be sustained for one hour (Gavin et al., 2012). FTP field tests are used to create a baseline from which the coach is able to assess the fitness of the athlete. Testing protocols range in duration from 20 minutes to 60 minutes. In these protocols athletes perform a maximum sustainable effort for the predetermined duration and mean power output is used to calculate FTP (Allen \& Coggan; 2010) There has been little investigation into how the testing environment may affect the results of such tests.

The exercise testing of athletes is usually performed in one of two environments: 1) outdoors in "real-world/on road" conditions, in which variables such as air temperature, wind, and gradient changes are similar to racing or training situations, and 2) indoors on stationary ergo-trainers, where conditions provide a consistent repeatable environment that does not have weather, traffic, or safety concerns. However, testing environments and lab conditions the use of fans is inconsistent and not always well documented potentially causing athletes, coaches, or investigators to unknowingly collect invalid results. Previous research in extreme thermal environments $\left(>35^{\circ} \mathrm{C}\right)$ has shown that fans can significantly affect results of laboratory tests. 
Due to variation in protocols and scarcity of validated testing protocols for most commonly used tests to measure FTP, for the purposes of this investigation time trial performance was used. In both running and cycling time trial tests have been shown to have high validity and are easily repeated (Laursen et al, 2007). Time trial tests are used as a valid measure of performance to simulate the real world environment and competition (Jeukendrup et al., 1996). Time trial procedures allow participants to ride at a self-selected pace rather than a defined power output, as long as the desired distance is covered as fast as possible. Time trial distance for this study was determined to be similar to the distances athletes from the test population would cover during common FTP test protocols (20-60 minutes).

Exercise is an exothermic activity, and as the exercise duration increases, an exercising individual can expect a predictable rise in body temperature. Core temperature can rise by $1^{\circ} \mathrm{C}$ per 5-7 minute interval (Kay et al., 1999). High body temperature and heat storage have been shown to have negative effects on exercise and sports performance (Ely et al. 2007). Precooling strategies — such as the use of ice vests-have been shown to have ergogenic effects on performance, countering the effects of increased body temperature by slowing or delaying the rise in body temperature (Marsh et al., 1999). Increased heart rate, decreased power output or pace, and increased rating of perceived exertion (RPE) have been observed with increased temperature (Hartley et al., 2011). Decreased efferent neural drive has been hypothesized as a potential limiting factor of exercise performance (Nybo et al., 2012). 
Current practices may or may not be to include a fan during tests but rarely is the magnitude of fan speed measured, recorded, or standardized. Current research investigating the use of forced air cooling by fans in indoor cycling has examined the effects under relatively extreme temperatures Adams et al. (1992) found that air flow of $3.5 \mathrm{~m} / \mathrm{s}$ was sufficient to attenuate an increase in esophageal temperature in $35^{\circ} \mathrm{C}$ laboratory conditions. An increase in sweat rate was also observed in $24^{\circ} \mathrm{C}$ and $35^{\circ} \mathrm{C}$ in still-wind conditions. Brown and Bannister (1985) sought to investigate differences in cardiovascular and thermoregulatory responses in standard lab, simulated outdoor, and outdoor cycling conditions. Simulated outdoor conditions were created using a combination of fans and lamps. The results show that performance in standard lab conditions with no air flow over the cyclist's body may impair performance. Mieras et al. (2014) built upon Brown and Bannister's work to include measurement of the psychological effects of outdoor and simulated outdoor cycling time trials. The findings suggest that athletes must work at a greater perceived effort to achieve the same results indoor as they would by training in an outdoor environment.

Saunders et al. (2005) in an investigation of fluid ingestion in hot humid environments with variable wind speeds used high speed fans to replicate air currents experienced at normal bicycling speeds. In hot environmental conditions it was observed thermoregulation was different in still wind conditions than those with cycling speed air velocities. Greater wind speeds resulted in lower ratings of heat storage, body temperature, and rating of perceived exertion. Significant differences in RPE were 
observed in the low fan $(10 \mathrm{~km} / \mathrm{hr})$ and control $(0 \mathrm{~km} / \mathrm{hr})$ conditions in comparison to higher wind speeds tested $(33-50 \mathrm{~km} / \mathrm{hr})$.

Fan cooling in stationary or simulated cycling has been shown to slow the rise in body temperature and reduce sweat rate. This investigation was designed as a pilot study to determine whether the effects observed in hot environments are transferable or relevant to athletes testing or training in standard room temperature environments.

The primary aim of this study was to investigate if the use of a fans to cool athletes in indoor or laboratory cycling test environment at "room temperature" (20$23^{\circ} \mathrm{C}$ ) affects intermediate duration performance test results. A secondary aim of this study was to investigate if differences exist for fan types readily available to athletes, coaches, and investigators. To achieve this, participants underwent three test conditions: a control and two experimental conditions. In one experimental condition a commercially available box fan was used to create a gentle wind cooling. Designed to replicate what is commonly used by cyclists testing themselves or in a limited lab conditions where higher output fans are too costly or impractical. The other experimental condition utilized a larger 48 inch industrial drum fan. This type of fan would still be readily available to consumers and creates higher air velocities over a larger area that may act in a similar manner to airflow created by locomotion. 


\section{Methods}

\section{Participants}

Eight experienced male competitive cyclists were recruited from the local Portland, Oregon metropolitan area; seven completed all trials and one was lost to attrition. Participants had a minimum of one year of racing experience as well as familiarity with stationary cycling trainers. Participant mean age of $28.5 \pm 2.45$ years, body mass of $70.3 \pm 9.45 \mathrm{~kg}$ and height of $174 \pm 12.7 \mathrm{~cm}$. Participants were provided Institutional Review Board-approved informed consent and Physical Activity Readiness Questionnaire (PAR-Q) before participation.

\section{Procedures}

Participants performed three $20-\mathrm{km}$ time trials, once for each test condition. Two experimental conditions were performed in addition to a control "no fan" condition: 1) a low speed fan condition and a high speed fan condition. A randomized cross-over design was used, with test condition sequence randomly drawn at the intake to the study. Participants provided their own bicycles to ensure familiarity of fit, positioning, and functioning of equipment. Participants were asked to keep a food journal for 24-hours prior to their first session and asked to replicate that diet as closely as possible before each subsequent test. Additionally, participants were asked to not make significant changes in their training or perform heavy exertions within 48 hours of each test. Each successive test took place within 2-5 days to minimize training and/or fatigue effects. 
Speed, distance and power output were measured using a Cycleops Hammer Direct-Drive Bicycle Trainer (Saris Corporation; Madison, WI, USA). The trainer was configured to allow participants to use their own bicycles. The direct-drive bicycle trainer was attached directly to the drivetrain of each participant's bicycle to directly measure power output with no significant loss to the tire-trainer interface. The manufacturer claims $\pm 3 \%$ accuracy in power measurements. Heart rate data were collected with a Bontrager chestmounted heart rate strap (Trek Bicycle Corp; Waterloo, WI, USA). All data were collected and compiled using the open source Golden Cheetah training software. Distance and speed were simulated based on power output for a $700 \times 25 \mathrm{~mm}$ road bicycle tire. Fan speeds were verified before each trial with a handheld Kestrel 3000 anemometer (Nielsen-Kellerman; Boothwyn, PA, USA).

All testing took place in the exercise physiology laboratory at Portland State University. Temperature was recorded before each test (mean temperature: $23.2 \pm$ $\left.1.63^{\circ} \mathrm{C}\right)$

The low fan speed experimental condition placed the fan at a distance of one meter in front of the bicycle. The fan was positioned on a platform $73 \mathrm{~cm}$ above the ground. A commercially available 20 inch $(50.8 \mathrm{~cm})$ box fan was used (Lasko Products; West Chester, PA, USA). Fan speed measured directly below participants' chins in this condition was $2.37 \pm 0.46 \mathrm{~m} / \mathrm{s}$. The high speed fan condition used a 48-inch $(1.22 \mathrm{~m})$ fan (Global Industrial, Port Washington, NY, USA) with an airflow of $19,500 \mathrm{ft}^{3} / \mathrm{min}(9.203$ $\mathrm{m}^{3} / \mathrm{s}$ ) produced by the front grill of the fan and was placed approximately one meter in 
front of the participant's bicycle. Fan speeds of $4.17 \pm 0.30 \mathrm{~m} / \mathrm{s}$ were measured in this experimental condition.

Before each test, the height and weight of each participant were measured. Participants then performed a 10-minute self-paced warm up with no fans in use. Following the warm-up, participants were given a 10-second countdown before the onset of the time-trial. In the two experimental conditions, fans were started during the countdown period. Participants were self-paced through all trials and were not provided with any verbal encouragement. During each test, participants were provided with verbal updates on their progress at 5, 10,15, and 19 kilometers. Participants were allowed ad libitum access to water during all tests. At the 10-kilometer and 19-kilometer points of each test, participants were asked to give a rating of perceived exertion (RPE) based on the original 6-20 point Borg RPE scale. At the conclusion of each trial, participants were given 5 minutes to cool down, after which body weight was measured. At the completion of all time trials, participants were provided with the results of each test.

\section{Statistical Analysis}

Data collected for analysis included time-trial time to completion, mean heart rate throughout the test, mean power output throughout the test, and ratings of perceived exertion. A subjects-by-trial repeated measures analysis of variance (ANOVA) was used to examine trial-to-trial data using a p-level of 0.05 to denote statistical significance. 


\section{Results}

\section{Time Trial Performance}

As seen in Figure 1, a modest increase in $20 \mathrm{~km}$ time trial performance appeared to occur in the experimental fan conditions over those of the control condition. Mean time to completion decreased $2.1 \%$ in the low speed fan condition $(2216 \mathrm{~s} \pm 58 \mathrm{~s}$ vs. 2262 $\mathrm{s} \pm 29 \mathrm{~s})$. The high speed fan condition resulted in a $3.3 \%$ decrease in time to completion (2190 s $\pm 67 \mathrm{~s})$. Results of time to completion were not statistically significant $(\mathrm{p}=0.06)$.

A $1.2 \%$ difference in mean time to completion was observed between the two experimental fan conditions.

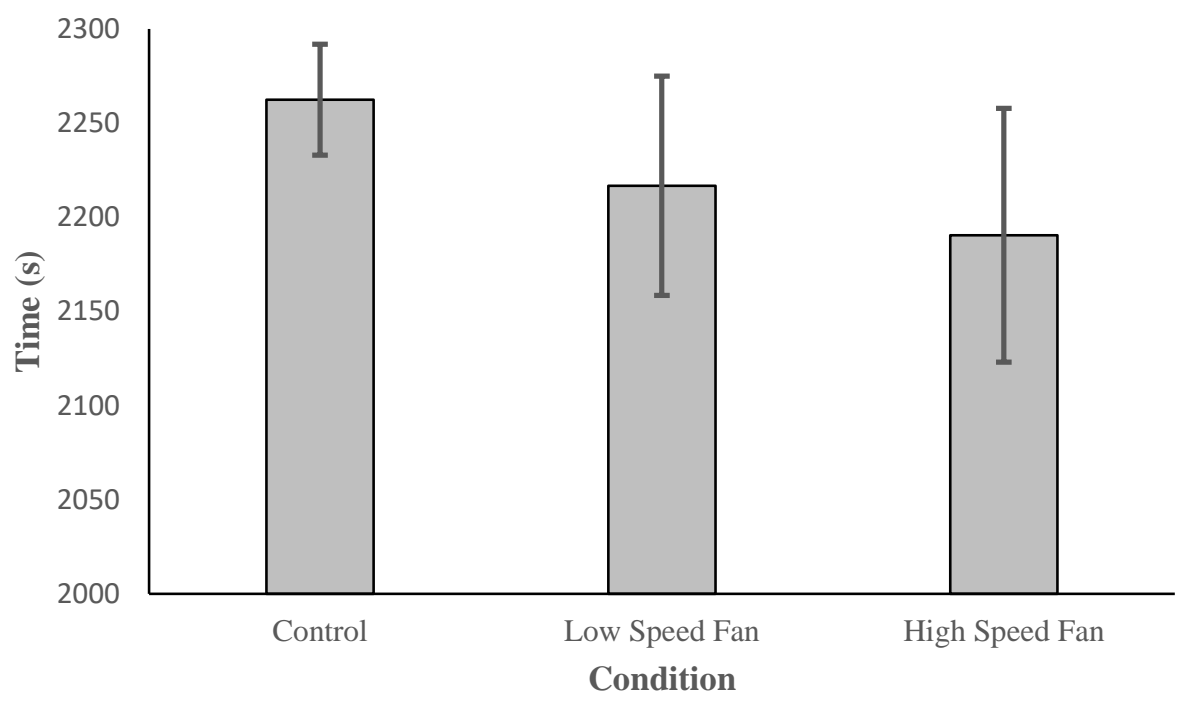

Figure 1. Mean time to completion for each test condition ( \pm SD) 
Figure 2 show that results of the experimental fan conditions were not consistent for all participants. One participant did not show any change in time to completion across all three conditions.

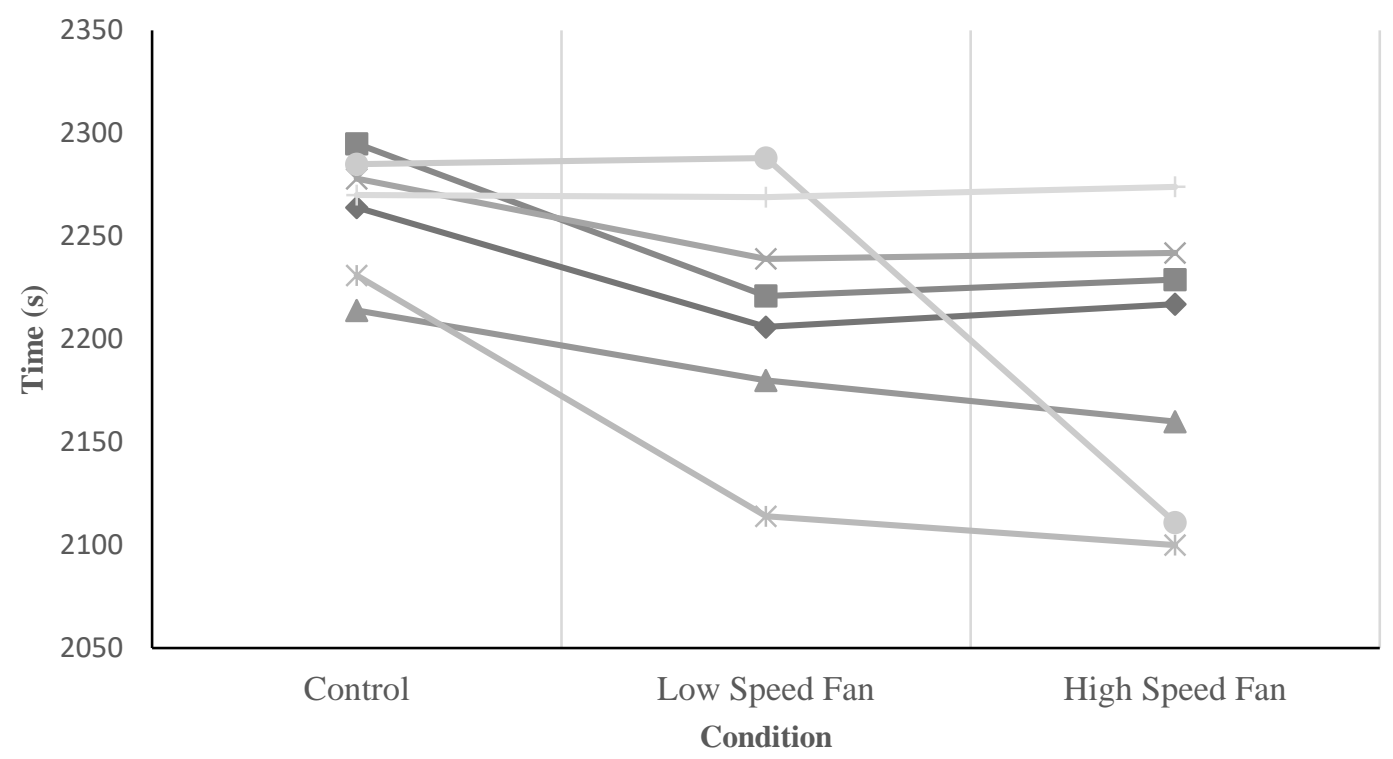

Figure 2. Time to completion across all conditions for all participants 


\section{Rating of Perceived Exertion}

Figure 3 shows that at $10 \mathrm{~km}$ there was no significant difference observed in RPE reported among trials $(\mathrm{p}=0.27)$. The average RPE reported during the control condition was $15.1 \pm 1.8$. A slight non-significant decrease in RPE appeared to occur in the experimental fan conditions. The mean low speed fan condition RPE was $14.4 \pm 1.1$,

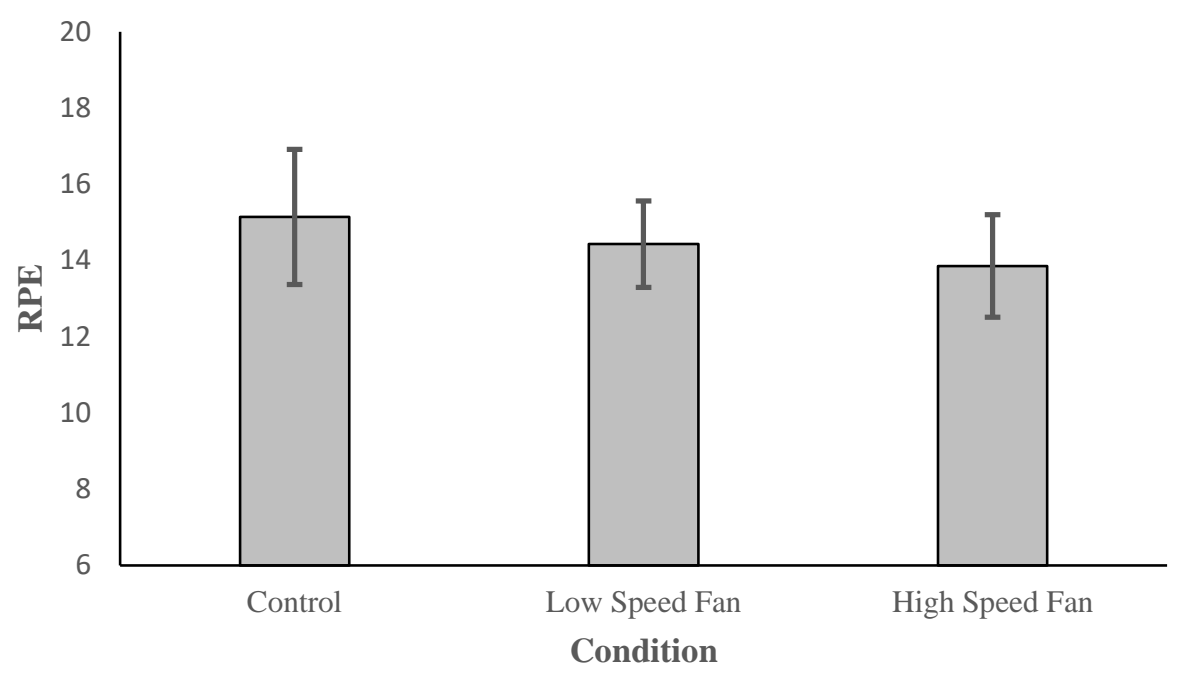

Figure 3. Mean $10 \mathrm{~km}$ RPE for each test condition $( \pm S D)$

while the mean high speed fan condition RPE was $13.9 \pm 1.3$. 
$19 \mathrm{~km}$ mean RPE results were similar to those at $10 \mathrm{~km}$. Figure 4 shows the mean

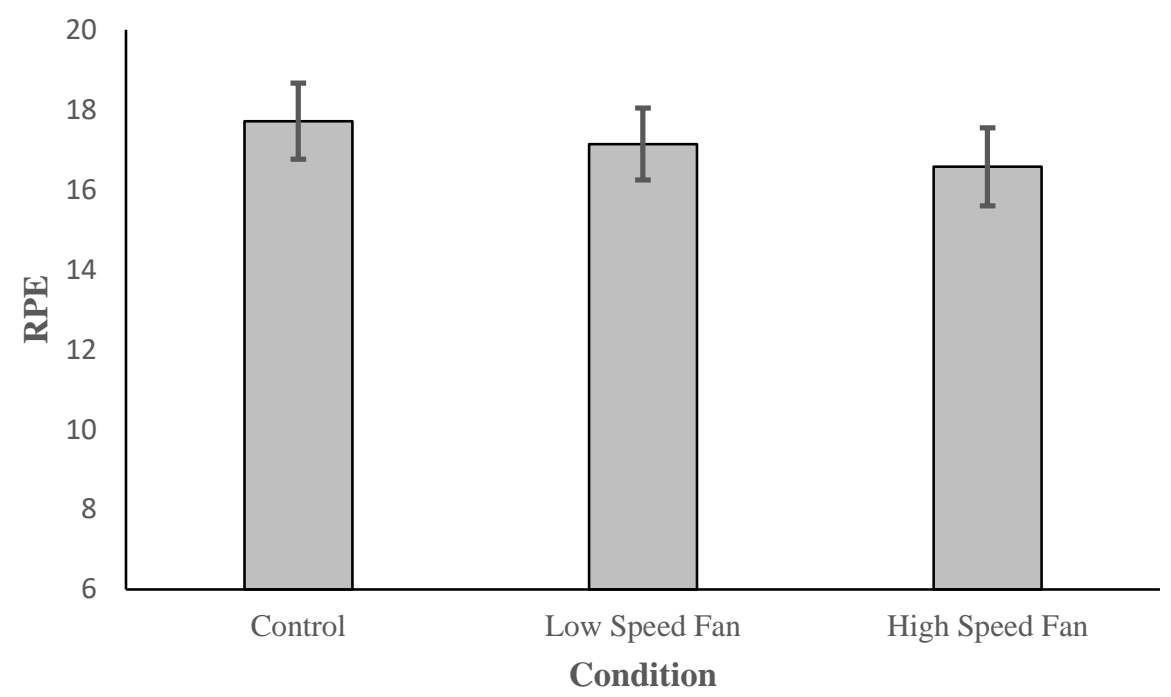

Figure 4. Mean $19 \mathrm{~km}$ RPE for each test condition $( \pm S D)$

RPE at the beginning of the final kilometer of the each trial was $17.7 \pm 0.9$ in the control condition, $17.1 \pm 0.9$ in the low speed fan condition and $16.5 \pm 1.0$ in the high speed fan condition. The apparent decrease in mean RPE was not found to be statistically significant $(\mathrm{p}=0.10)$.

\section{Power Output}

Mean power output appeared to increase slightly in the experimental fan conditions versus the control condition. Mean power output differences over the control 


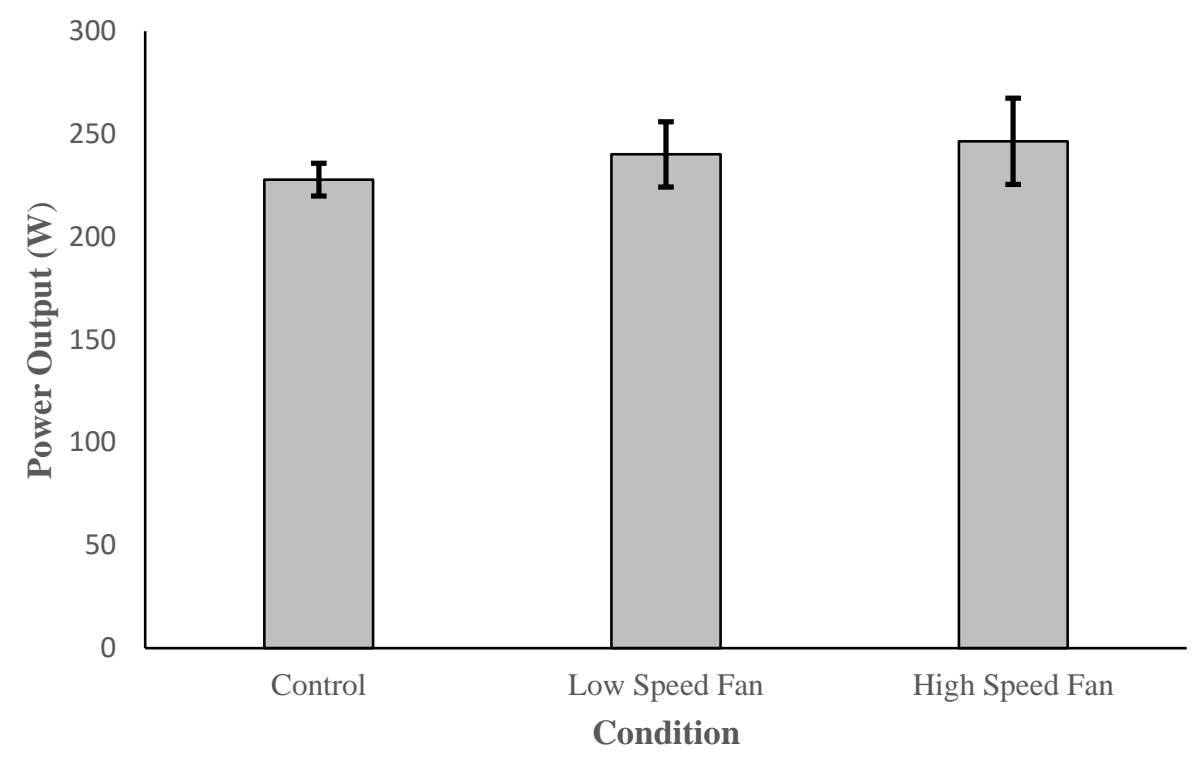

Figure 5. Mean power output for each test condition $( \pm S D)$

condition were $5.1 \%$ and $7.6 \%$ in to the low and high speed fan conditions, respectively. Mean power outputs are shown in Figure 5: $228 \mathrm{~W} \pm 8 \mathrm{~W}$ in the control condition, $240 \mathrm{~W}$ $\pm 16 \mathrm{~W}$ in the low speed fan condition, $246 \mathrm{~W} \pm 21 \mathrm{~W}$ in the high speed fan condition. Differences in mean power output were not statistically significant $(p=0.06)$ 


\section{Heart Rate}

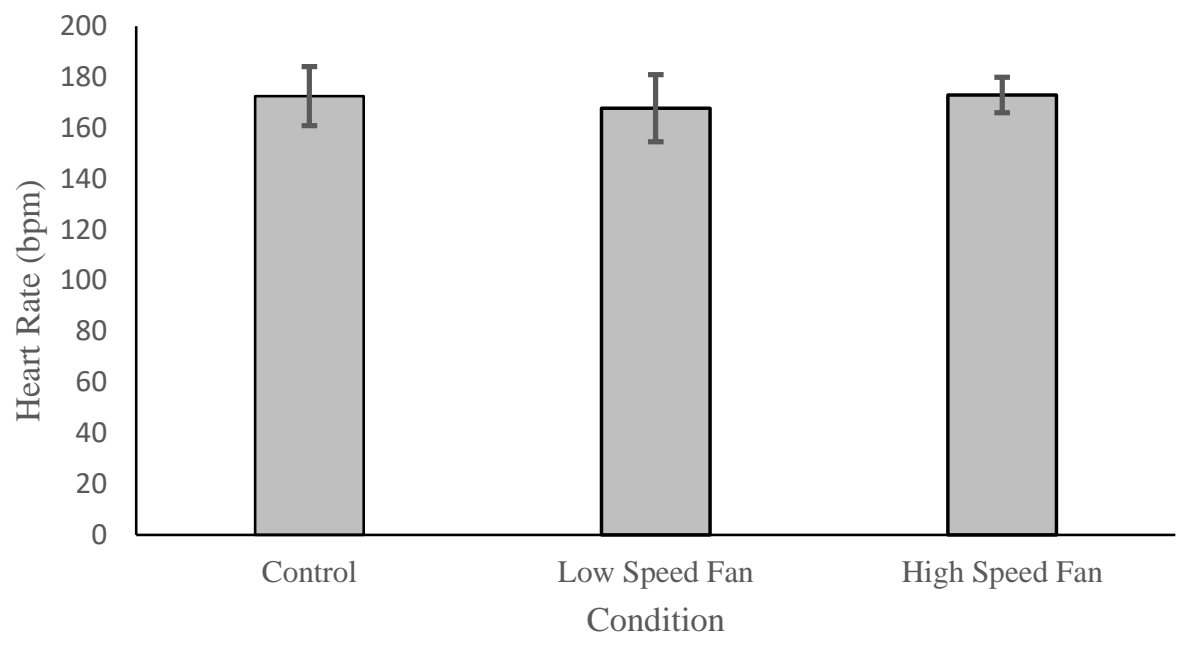

Figure 6. Mean heart rate for each test condition $( \pm S D)$

No statistically significant differences were observed in mean heart rate for any of the conditions $(\mathrm{p}=0.62)$. Mean heart rates were $172 \mathrm{bpm} \pm 12 \mathrm{bpm}, 168 \mathrm{bpm} \pm 13 \mathrm{bpm}$, and $173 \mathrm{bpm} \pm 7 \mathrm{bpm}$ in the control, low speed fan, and high speed fan conditions, respectively (Figure 6). 


\section{Discussion}

Although the results of time trial time to completion did not reveal a statistically significant difference between fan conditions and control, it appeared that a small effect may exist. The majority of participants demonstrated a reduction in time to completion in one or both of the experimental conditions. One subject produced consistent time trial performances, differences in experimental condition times to completion for this subject were less than $0.25 \%$ of those observed in the control condition (low speed fan $-0.04 \%$; high speed fan $+0.17 \%)$. A statistically significant difference among conditions is revealed when this subject's data are removed from the analysis $(p=0.04)$, but the reduction in sample size limits the extent to which generalizable conclusions can be drawn. Also, the relatively small sample size used in this study—combined with the large variation among subjects—-resulted in an increased probability of a Type II error. Any effect of fan use during indoor time trial performance at room temperature is likely to be small (reduction in mean times ranged from $2.1 \%$ to $3.3 \%$ ). The difference in mean time to completion between the two experimental conditions was only $1.2 \%$. These findings are supported by the work of Adams and colleagues (1992) who found fan speeds as low as $3.5 \mathrm{~m} / \mathrm{s}$ were sufficient to elicit improvements in performance. Fan speeds for this investigation ranged $2.4-4.2 \mathrm{~m} / \mathrm{s}$ in the experimental conditions. Additionally, the small difference in mean time to completion between the two experimental conditions suggests greater fan speeds likely do not have any ergogenic effects beyond the modest improvements observed in the low speed fan condition. 
Mean power output was observed to increase slightly in both experimental conditions (5.1\% and 7.6\% for low speed fan and high speed fan, respectively). Although not statistically significant, an increase in mean power output was expected, as time trial performance (time to completion) is a direct result of power output when external forces such as wind or changes in gradient are controlled. A difference of $2.54 \%$ was observed between the low speed fan and high speed fan conditions power outputs between the two experimental fan conditions. Taking measurement error into account, there is likely no difference in the power output of the two experimental conditions as observed in this investigation. This again suggests that increased fan speeds have no measurable performance advantage over low fan speeds.

Mean ratings of perceived exertion at both $10-\mathrm{km}$ and $19-\mathrm{km}$ were not significantly different between the experimental conditions. Several participants notedregardless of their time trial performance - the control condition was more distressing psychologically and more uncomfortable than either of the experimental fan conditions. Despite the feedback regarding participant comfort, there was very little difference in RPE between any of the conditions. It should be noted that all but one participant reduced time trial time and small increase in mean power output in at least one of the experimental conditions versus the control condition. Previous studies have been inconsistent in establishing a link between airflow and RPE. Saunders et al. (2004) observed a significantly lower RPE reported while cycling in extreme $35^{\circ} \mathrm{C}$ with a fan than without. Mieras et al. (2014) observed no difference in RPE for still-air laboratory conditions versus simulated and actual outdoor cycling. Similarly RPE was not 
significantly different between conditions in this investigation In both this investigation and the investigation by Mieras and colleagues (2014) participants were told to ride at the same relative effort through all trials. Saunders and colleagues (2004) required participants to work at a fix rate as determined by a percentage of $\mathrm{VO}_{2}$ max. This suggests RPE may be more sensitive when working at a fixed load rather than a self-selected effort.

Participants preferred both experimental conditions were preferred by participants over the control condition. At the time of the current study no metric was used to quantitatively measure comfort/discomfort as it related to the environment conditions. It was hypothesized that any change in comfort/discomfort would be reflected in RPE. This appears not to be the case. It was hypothesized that the low speed fan and control conditions would be consistent with previous studies conducted in the heat, with participants reporting greater RPE in the control condition. The results of this study did not support the hypothesis. Most of the participants in the current study were able to generate a higher power output in the experimental conditions than the control condition at the same RPE. These results show potential for increased power at the same selfselected intensity. The potential for increased power output, if significant, would allow athletes to train at higher intensities in an indoor setting.

Mean heart rate was not significantly different among conditions. Increased body temperature or heat exposure has been shown to increase heart rate during exercise; a phenomenon termed "cardiovascular drift." Cardiovascular drift occurs as a result of fluid loss and redistribution of blood volume to subcutaneous capillary beds reducing venous 
return and atrial filling (Jeukendrup \& Diemen, 1998). It is likely that the 20-km time trial tests may not have been long enough to elicit the effects of cardiovascular drift often observed in longer duration exercise in extreme heat. Assuming that the room temperature was not sufficient to induce sufficient thermal stress, it is not surprising that the consistency in heart rate data are similar to that seen in RPE. The majority of participants demonstrated faster $20 \mathrm{~km}$ time trials in the experimental conditions, while heart rates were similar to the control condition.

The consistency and low variability in the control condition suggests that the sample of participants was homogenous. All participants in the current study had at least one year of competitive cycling experience as well as familiarity with stationary ergotrainers. This homogeneity suggests that differences in training status or fitness levels are not likely to explain the variance observed in the experimental conditions. The variability in the responses to the experimental conditions suggest that any beneficial effects of fan use during indoor time trial performance may not be the same for everyone. Further investigation is needed to determine what individual factors may influence the response to airflow in time trial performance.

The results of this study offer support for the use of fans-even small consumer models - to create airflow for improved comfort, and may result in small benefits in performance or training intensity. It may be advisable to recommend that athletes testing and training at room temperature use fans for comfort, even if there is little effect on overall performance. This is supported by previous studies such as those by Adams et al. (1992), where air velocities as low as $3.5 \mathrm{~m} / \mathrm{s}$ were found to be sufficient to attenuate the 
rise in body temperature associated with exercise in a $35^{\circ} \mathrm{C}$ environment. The use of either fan used in the current study did not have any detrimental effects, and complaints of discomfort in the control condition appears to further support their use.

\section{Limitations}

This investigation examined cycling performance in an indoor stationary cycling environment. The results of this study should not be extrapolated to outdoor or nonstationary modes of testing. Indoor cycling can be well-controlled, and confounding environmental influences are minimized, whereas outdoor cycling includes numerous environmental factors that may influence results. These differences affect the ecological validity and should be considered when interpreting the results of this study.

Constraints on recruitment and the significant time investment required of participants resulted in a limited sample size. Participants were required to transport their bicycles to the lab a minimum of three times in order to participate in the study. The three testing sessions averaged approximately one hour each, and the fact that volunteers were not compensated for their travel and time had a negative effect on recruitment.

It is important to acknowledge that body temperature was not measured in this study, and conclusions about the effects of fan cooling on core temperature during indoor cycling are therefore not possible. However, logistical, recruitment, and financial considerations limited our ability to include core body temperature as a dependent 
variable, and we focused our interest on time-trial performance, heart rate, and perceptual responses to investigate the effects of fan cooling.

The study was conducted in late winter and early spring at the beginning of the competitive cycling season in the region. Therefore, the training status of participants was varied and uncontrolled (i.e., athletes with early season goals entered the study with a higher level of overall fitness). Many were involved in strenuous training at the beginning of the study, and an examination of each athletes' individual results indicated that at least some of the participants' results may have been affected by training prior to or during participation in this investigation.

\section{Further Inquiry}

A larger sample of participants is needed to more accurately investigate the effects of forced air cooling during indoor cycling time trial tests. A larger sample would better be able to provide evidence to determine whether the results observed in one participant of this study represent an outlier. This is important to determine if individual differences play a role in the response to forced air cooling.

Test duration or distance may also have an effect. The scope of this investigation was for short duration efforts. Previous studies typically used longer efforts of an hour or $40 \mathrm{~km}$. The rise in body temperature is known to be a product of time as demonstrated by Kay and colleagues (1999). A similar study design with a longer duration of $40 \mathrm{~km}$ could allow greater time for significant differences to become apparent. The results of this investigation suggest that if fan use has effects on time trial performance, they are small. 
The distance tested in this study may not have been sufficient to elicit the effects hypothesized.

A review of the published studies related to the effects of airflow on cycling performance provides little information about the placement of fans relative to the athlete. Localizing airflow to specific regions of the body could be beneficial, as different regions exhibit varying degrees of vascularization and density of capillary beds and sweat glands. Likewise, the psychological effects of wind or fan direction should also be investigated. In sports such as running or cycling, movement into a headwind (airflow opposing the direction of travel) has significant negative effects in real world environments. In contrast, a tailwind (airflow originating from behind the athlete) can have a positive influence on speed and performance. The potential for a psychological benefit (placebo effect) also exists. Previous studies have shown that a suggestion that a treatment or condition has positive effects can increase performance. 


\section{Conclusions}

Overall, no detrimental effects on performance were observed with the use of a fan in short duration cycling performance tests. Trends for small improvements in time to completion and increased power output were observed. The effect of the room temperature environment may not, even in still air conditions, create enough heat stress that increased fan speed would result in any additional benefit. At room temperature, excess heat is lost at a higher rate than in a hot environments $\left(>30^{\circ} \mathrm{C}\right)$. Previous studies of fan cooling have been undertaken in higher temperature conditions, resulting in significantly greater heat stress.

Fans may be beneficial for the psychological well-being and comfort of participants undergoing training or performance testing at room temperature. Based on statements made by study participants, the use of a fan - at any fan speed — is preferable and less distressing. Several participants noted they felt significantly more uncomfortable and found the effort more stressful in the control condition, despite no significant difference in RPE. A link may yet exist between indoor time trial performance and the use of fan cooling, but the results of this investigation are inconclusive. Many of the results suggest modest improvements in performance, but the large variances observed suggest individual responses differ greatly.

The effects of fan use at room temperature appear to be small, and more thorough investigation is needed. The lack of any detriments and the improved comfort of athletes 
undergoing performance tests should be enough to recommend the use of a fan.

Additionally, the performance of some athletes appeared to benefit from the presence of a fan. 


\section{References}

Adams, W. C., Mack, G. W., Langhans, G. W., \& Nadel, E. R. (1992). Effects of varied air

velocity on sweating and evaporative rates during exercise. Journal of Applied Physiology,73(6), 2668-2674. doi:10.1152/jappl.1992.73.6.2668

Allen, H., \& Coggan, A. (2010). Training and racing with a power meter. Boulder, Co.: Velopress.

Altareki, N., Drust, B., Atkinson, G., Cable, T., \& Gregson, W. (2009). Effects of environmental

heat stress $\left(35^{\circ} \mathrm{C}\right)$ with simulated air movement on the thermoregulatory responses during a 4-km cycling time trial. International Journal of Sports Medicine, 30 (1), 9-15.

Brown, S. L., \& Banister, E. W. (1985). Thermoregulation during prolonged actual and laboratory-simulated bicycling. European Journal of Applied Physiology and Occupational Physiology, 54(1), 125-130.

Ely, M. R., Cheuvront, S. N., Roberts, W. O., \& Montain, S. J. (2007). Impact of weather on

marathon-running performance. Medicine \& Science in Sports \& Exercise, 39 (3), 487-493. doi:10.1249/mss.0b013e31802d3aba

Gavin, T. P., Meter, J. B., Brophy, P., Dubis, G., Potts, K. N., \& Hickner, R. C. (2011). 
Comparison of a field based test to estimate functional threshold power and power output at lactate threshold. Medicine \& Science in Sports \& Exercise,43 (Suppl 1), 625-626. doi:10.1249/01.mss.0000401727.75886.7a

Hartley, G. L., Flouris, A. D., Plyley, M. J., \& Cheung, S. S. (2012). The effect of a covert

manipulation of ambient temperature on heat storage and voluntary exercise intensity. Physiology \& Behavior, 105(5), 1194-1201.

Jeukendrup, A., Saris, W. H. M., Brouns, F., \& Kester, A. D. (1996). A new validated endurance

performance test. Medicine and Science in Sports and Exercise, 28(2), 266-270.

Jeukendrup, A., \& Diemen, A. V. (1998). Heart rate monitoring during training and competition

in cyclists. Journal of Sports Sciences, 16 (Sup1), 91-99.

doi:10.1080/026404198366722

Kay, D., Taaffe, D. R., \& Marino, F. E. (1999). Whole-body pre-cooling and heat storage during

self-paced cycling performance in warm humid conditions. Journal of Sports Sciences, 17 (12), 937-944.

Laursen, P. B., Francis, G. T., Abbiss, C. R., Newton, M. J., \& Nosaka, K. (2007). Reliability of time-to-exhaustion versus time-trial running tests in runners. Medicine \& Science in Sports \& Exercise, (39), 1374-9. 
Marsh, D., \& Sleivert, G. (1999). Effect of precooling on high intensity cycling performance. British Journal of Sports Medicine, 33(6), 393-397.

Mieras, M. E., Heesch, M. W., \& Slivka, D. R. (2014). Physiological and psychological responses to outdoor vs. laboratory cycling. The Journal of Strength \& Conditioning Research, 28 (8), 2324-2329.

Nybo, L. (2012). Brain temperature and exercise performance. Experimental Physiology, 97 (3), 333-339.

Peiffer, J. J., \& Abbiss, C. R. (2011). Influence of environmental temperature on $40 \mathrm{~km}$ cycling

time-trial performance. International Journal of Sports Physiology and Performance,6 (2), 208-220. doi:10.1123/ijspp.6.2.208

Saunders, A. G., Dugas, J. P., Tucker, R., Lambert, M. I., \& Noakes, T. D. (2005). The effects of different air velocities on heat storage and body temperature in humans cycling in a hot, humid environment. Acta Physiologica Scandinavica, 183 (3), 241-255.

Shaffrath, J. D., \& Adams, W. C. (1984). Effects of airflow and work load on cardiovascular drift and skin blood flow. Journal of Applied Physiology, 56 (5), 1411-1417. Smith, J. C., Dangelmaier, B. S., \& Hill, D. W. (1999) Critical power is related to cycling time

trial performance. International Journal of Sports Medicine, 20, 374-378. 